\title{
The Seiberg-Witten Map for Noncommutative Gauge Theories
}

\author{
B. L. Cerchiai*, A. F. Pasqua ${ }^{\dagger}$, B. Zumino ${ }^{\ddagger}$
}

\author{
Department of Physics \\ University of California \\ and \\ Theoretical Physics Group \\ Lawrence Berkeley National Laboratory \\ University of California \\ Berkeley, California 94720
}

\begin{abstract}
The Seiberg-Witten map for noncommutative Yang-Mills theories is studied and methods for its explicit construction are discussed which are valid for any gauge group. In particular the use of the evolution equation is described in some detail and its relation to the cohomological approach is elucidated. Cohomological methods which are applicable to gauge theories requiring the Batalin-Vilkoviskii antifield formalism are briefly mentioned. Also, the analogy of the Weyl-Moyal star product with the star product of open bosonic string field theory and possible ramifications of this analogy are briefly mentioned.
\end{abstract}

\footnotetext{
*email address: BLCerchiai@lbl.gov

†email address: pasqua@socrates.berkeley.edu

‡email address: zumino@thsrv.lbl.gov
} 


\section{Introduction}

Noncommutative field theories have recently received much attention. Seiberg and Witten [1] have argued that certain noncommutative gauge theories are equivalent to commutative ones and in particular that there exists a map from a commutative gauge field to a noncommutative one, which is compatible with the gauge structure of each. This map has become known as the Seiberg-Witten (SW) map.

In two recent papers $[2,3]$ we have discussed a cohomological method for constructing explicitly this map. Here we describe a slightly modified procedure based on the idea that the structure equations of the gauge group of the noncommutative theory are a deformation of those of the gauge group of the commutative theory. We will consider gauge theories on the noncommutative space defined by

$$
\left[x^{i}, x^{j}\right]=i \theta^{i j},
$$

where $\theta$ is a constant Poisson tensor. The " $\star$ " operation is the associative Weyl-Moyal product

$$
f \star g=f e^{\frac{i}{2} \theta^{i j} \overleftarrow{\partial_{i}} \overrightarrow{\partial_{j}}} g
$$

We believe that our methods are much more general, and can in fact be used even when $\theta$ is not constant, but in this paper we shall make use of the fact that the $x^{i}$ derivative $\partial_{i}$ of functions satisfies the Leibniz rule with respect to the star product

$$
\partial_{i}(f \star g)=\left(\partial_{i} f\right) \star g+f \star\left(\partial_{i} g\right),
$$

just as it does with respect to the ordinary product. This simple relation requires $\theta$ to be constant.

\section{Structure Equations}

The structure equations of a gauge group can be expressed in terms of a ghost field $\lambda(x)$ and the gauge potential $a_{i}(x)$ by giving the action of the BRST operator $s$

$$
\begin{aligned}
s \lambda & =i \lambda \cdot \lambda, \\
s a_{i} & =\partial_{i} \lambda-i a_{i} \cdot \lambda+i \lambda \cdot a_{i} .
\end{aligned}
$$

Here $\lambda$ and $a_{i}$ are valued in a Lie algebra and can be represented by matrices, the matrix elements of the ghost field being anticommuting functions of $x$. In a representation the product would imply matrix multiplication. The operator $s$ is an odd superderivation of ghost number one

$$
\begin{aligned}
s(f \cdot g) & =(s f) \cdot g \pm f \cdot s g \\
s^{2} & =0
\end{aligned}
$$


which commutes with the derivatives

$$
s \partial_{i}=\partial_{i} s .
$$

As usual, the signs in (6) depend on the parity of $f$. Our task is to deform the above structure equations into

$$
\begin{aligned}
s \Lambda & =i \Lambda \star \Lambda, \\
s A_{i} & =\partial_{i} \Lambda-i\left[A_{i} \stackrel{\star}{,} \Lambda\right],
\end{aligned}
$$

where $A_{i}=A_{i}\left(a, \partial a, \partial^{2} a, \ldots\right)$ is an even local functional of $a_{i}$, of ghost number zero, and $\Lambda=\Lambda(\lambda, \partial \lambda, \ldots, a, \partial a, \ldots)$ is an odd local functional of $a_{i}$ and $\lambda$, of ghost number one (like $\lambda$ ). We take $s$ to be undeformed and to satisfy (7), (8) and

$$
s(f \star g)=s f \star g \pm f \star s g .
$$

The solution consists in finding explicit expressions for the functionals $A_{i}$ and $\Lambda$. This can be done as expansions in $\theta$

$$
\begin{aligned}
& \Lambda=\Lambda^{(0)}+\Lambda^{(1)}+\ldots, \quad \Lambda^{(0)}=\lambda, \\
& A_{i}=A_{i}^{(0)}+A_{i}^{(1)}+\ldots, \quad A_{i}^{(0)}=a_{i} .
\end{aligned}
$$

The first order terms were given already in [1]

$$
\begin{aligned}
\Lambda^{(1)} & =\frac{1}{4} \theta^{k l}\left\{\partial_{k} \lambda, a_{l}\right\}, \\
A_{i}^{(1)} & =-\frac{1}{4} \theta^{k l}\left\{a_{k}, \partial_{l} a_{i}+f_{l i}\right\},
\end{aligned}
$$

where

$$
f_{l i}=\partial_{l} a_{i}-\partial_{i} a_{l}-i\left[a_{l}, a_{i}\right]
$$

is the commutative field strength, and expressions for $\Lambda^{(2)}$ and $A_{i}^{(2)}$ are known $[2$, $4,6]$, see also below.

A systematic way to obtain the expansion in $\theta$ was described in $[2,3]$ and the consistency of the procedure was demonstrated in [7]. Each order in $\theta$ is manifestly local.

One sees already from (14) and (15) that $\Lambda$ and $A_{i}$ cannot be Lie algebra valued in general, and we follow $[5,6]$ by allowing them to be in the enveloping algebra of the Lie algebra of $\lambda$ and $a_{i}$. A representation of this Lie algebra lifts naturally to a representation of its enveloping algebra.

\section{Evolution Equations}

There is an alternative approach for the study of the SW map, which is based on a differential equation [1]. Let us introduce a "time" parameter $t$ in front of $\theta$, in such a way that $\theta^{i j} \rightarrow t \theta^{i j}, \Lambda \rightarrow \Lambda(t)$ and $A_{i} \rightarrow A_{i}(t)$, while keeping 
$s$ independent of $t$. Notice that $\Lambda$ and $A_{i}$ acquire a $t$-dependence through $\theta$. Differentiating the structure equations (9) and (10) with respect to $t$, we obtain ${ }^{1}$

$$
\begin{aligned}
s \dot{\Lambda} & =i \dot{\Lambda} \star \Lambda+i \Lambda \star \dot{\Lambda}+i \Lambda \star \Lambda, \\
s \dot{A}_{i} & =\dot{A}_{i} \star \Lambda+\Lambda \star \dot{A}_{i}+D_{i} \dot{\Lambda}-i A_{i} \star \Lambda+i \Lambda \nless A_{i},
\end{aligned}
$$

where

$$
D_{i}=\partial_{i}-i\left[A_{i} \stackrel{\star}{,} \cdot\right]
$$

is the covariant derivative at time $t$. The star product itself depends on the evolution parameter $t$, and therefore it has also to be differentiated

$$
\star=e^{\frac{1}{2} i t \overleftarrow{\partial_{i}} \theta^{i j} \overrightarrow{\partial_{j}}}, \quad \dot{\star}=e^{\frac{1}{2} i t \overleftarrow{\partial_{i}} \theta^{i j}} \overrightarrow{\partial_{j}} \frac{i}{2} \overleftarrow{\partial_{k}} \theta^{k l} \overrightarrow{\partial_{l}} .
$$

Explicitly this yields

$$
f \dot{\star} g=i \frac{\theta^{k l}}{2} \partial_{k} f \star \partial_{l} g .
$$

Notice that for simplicity we have restricted ourselves to a linear path in $\theta$-space, i.e. we are considering a linear one-parameter family of deformations of $\theta$. In principle it would be possible to consider an arbitrary variation with respect to $\theta$ corresponding to an arbitrary path in $\theta$-space, like e.g. in [4].

The structure of the right hand side of (17) and (18) leads in a natural way to the definition of a new operator ${ }^{2}$ at time $t$ :

$$
\Delta_{t}=\left\{\begin{array}{cc}
s-i\left\{\Lambda_{,}^{\star} \cdot\right\} & \text { on odd quantities, } \\
s-i\left[\Lambda_{,}^{\star} \cdot\right] & \text { on even quantities. }
\end{array}\right.
$$

It has the following properties

$$
\begin{gathered}
\Delta_{t} A_{i}=\partial_{i} \Lambda, \quad \Delta_{t}^{2}=0, \quad\left[\Delta_{t}, D_{i}\right]=0, \\
\Delta_{t}\left(f_{1} \star f_{2}\right)=\left(\Delta_{t} f_{1}\right) \star f_{2} \pm f_{1} \star\left(\Delta_{t} f_{2}\right),
\end{gathered}
$$

i.e. $\Delta_{t}$ is nilpotent, it commutes with the covariant derivative at time $t$ and it satisfies a super-Leibniz rule. This is a consequence of the fact that

$$
s^{2}=0, \quad s \partial_{i}=\partial_{i} s,
$$

and of the associativity of the star product. Therefore, $\Delta_{t}$ can be interpreted as a coboundary operator in a suitably defined cohomology.

Using the operators $\Delta_{t}$ and $D_{i}$ the equations (17) and (18) can be rewritten as

$$
\begin{aligned}
\Delta_{t} \dot{\Lambda} & =-\frac{1}{2} \theta^{k l} \partial_{k} \Lambda \star \partial_{l} \Lambda=-\frac{1}{2} \theta^{k l} B_{k} \star B_{l}, \\
\Delta_{t} \dot{A}_{i} & =D_{i} \dot{\Lambda}+\frac{1}{2} \theta^{k l}\left\{\partial_{k} A_{i}, \partial_{l} \Lambda\right\}=D_{i} \dot{\Lambda}+\frac{1}{2} \theta^{k l}\left\{\partial_{k} A_{i} \stackrel{\star}{,} B_{l}\right\} .
\end{aligned}
$$

\footnotetext{
${ }^{1}$ As customary the dot denotes differentiation with respect to $t$.

${ }^{2} \Delta_{t}$ is a simple generalization of the operator $\Delta$ introduced in [2], which now should be called $\Delta_{0}$. Also, what was called $\hat{\Delta}$ in [3] should now be called $\Delta_{1}$.
} 
Here we have introduced the notation

$$
B_{i}=\partial_{i} \Lambda,
$$

which is useful because only derivatives of $\Lambda$ enter in the right hand side of (26) and (27), but never $\Lambda$ itself. The action of $\Delta_{t}$ in terms of these new variables $A_{k}$ and $B_{k}$ takes a particularly simple form

$$
\Delta_{t} A_{k}=B_{k}, \quad \Delta_{t} B_{k}=0 .
$$

With this action the consistency condition that $\Delta_{t}$ applied to the right hand side of equation (26) vanishes is verified. For (27) we find that $\Delta_{t}$ on the right hand side gives $\frac{1}{2} \theta^{k l}\left[\Delta_{t} F_{k i} \stackrel{\star}{,} B_{l}\right]$. We will comment on this later in section 4 .

The differential evolution equations which provide a solution to the equations (26), (27) are given by [1]

$$
\begin{aligned}
\dot{\Lambda} & =\frac{1}{4} \theta^{i j}\left\{\partial_{i} \Lambda \stackrel{\star}{,} A_{j}\right\}, \\
\dot{A}_{i} & =-\frac{1}{4} \theta^{k l}\left\{A_{k} \stackrel{\star}{,} \partial_{l} A_{i}+F_{l i}\right\},
\end{aligned}
$$

where

$$
F_{l i}=\partial_{l} A_{i}-\partial_{i} A_{l}-i\left[A_{l} \stackrel{\star}{,} A_{i}\right]
$$

is the noncommutative field strength. This can be easily checked by substituting these expressions in (26) and (27).

\section{The Homotopy Operator}

There is a way of computing the expressions (30) for $\dot{\Lambda}$ and (31) for $\dot{A}_{i}$ through a suitably defined homotopy operator $K_{t}$. Clearly, it is not possible to invert $\Delta_{t}$, because it is nilpotent, but if we construct an operator such that

$$
K_{t} \Delta_{t}+\Delta_{t} K_{t}=1
$$

then an equation of the form

$$
\Delta_{t} f=m,
$$

with

$$
\Delta_{t} m=0,
$$

has a solution of the type

$$
f=K_{t} m,
$$

because

$$
m=\Delta_{t} K_{t} m+K_{t} \Delta_{t} m=\Delta_{t} K_{t} m .
$$

The solution (36) is not unique: $K_{t} m+\Delta_{t} h$, with some appropriate $h$, is also a solution, since $\Delta_{t}^{2}=0$. This is the same method we applied for $t=0$ in 
$[2,3]$, which closely follows the ideas developed in [8] to study anomalies in chiral gauge theories.

Let us construct such a homotopy operator $K_{t}$ explicitly. We start by defining a linear operator $\tilde{K}_{t}$ such that

$$
\tilde{K}_{t} B_{k}=A_{k}, \quad \tilde{K}_{t} A_{k}=0
$$

On both $A_{k}$ and $B_{k}$ it satisfies

$$
\tilde{K}_{t} \Delta_{t}+\Delta_{t} \tilde{K}_{t}=1
$$

Further, we require that it is a super-derivation

$$
\tilde{K}_{t}\left(f_{1} \star f_{2}\right)=\left(\tilde{K}_{t} f_{1}\right) \star f_{2} \pm f_{1} \star\left(\tilde{K}_{t} f_{2}\right)
$$

and that it commutes with $D_{i}$ and anticommutes with $s$

$$
\left[\tilde{K}_{t}, D_{i}\right]=0, \quad\left\{\tilde{K}_{t}, s\right\}=0 .
$$

Notice that due to $(39) \tilde{K}_{t}$ has to be odd and it decreases the ghost number by one. Moreover, it is nilpotent on $A_{i}, B_{i}$

$$
\tilde{K}_{t}^{2}=0 \text {. }
$$

On monomials of higher order in $A_{k}$ and $B_{k}$, the homotopy operator $K_{t}$ cannot satisfy the Leibniz rule. If $d$ is the total order of such a monomial $m$, then the action of $K_{t}$ on it has to be defined as

$$
K_{t} m=d^{-1} \tilde{K}_{t} m
$$

It is extended to general polynomials by linearity. Then $K_{t}$ satisfies (33) and from (42) it follows that

$$
K_{t}^{2}=0 .
$$

Now, we can use $K_{t}$ to recover the solutions (30), (31) of the equations (26), (27). For $\Lambda$ this is straightforward. We apply $K_{t}$ to the right hand side of (26) and we get

$$
\begin{aligned}
\dot{\Lambda} & =K_{t}\left(-\frac{1}{2} \theta^{k l} B_{k} \star B_{l}\right)=-\frac{1}{4} \theta^{k l}\left(\tilde{K}_{t} B_{k} \star B_{l}-B_{k} \star \tilde{K}_{t} B_{l}\right) \\
& =-\frac{1}{4} \theta^{k l}\left(A_{k} \star B_{l}-B_{k} \star A_{l}\right)=\frac{1}{4} \theta^{k l}\left\{B_{k} \star A_{l}\right\},
\end{aligned}
$$

which coincides with (30).

For the gauge potential, however, there is a complication. If we apply $\Delta_{t}$ to the right hand side of (27) we obtain

$$
\Delta_{t}\left(D_{i} \dot{\Lambda}+\frac{1}{2} \theta^{k l}\left\{\partial_{k} A_{i} \stackrel{\star}{,} B_{l}\right\}\right)=\frac{1}{2} \theta^{k l}\left[\Delta_{t} F_{k i} \stackrel{\star}{,} B_{l}\right],
$$


where $\Delta_{t} F_{k i}=D_{k} B_{i}-D_{i} B_{k}+i\left[B_{k}, A_{i}\right]+i\left[A_{k}, B_{i}\right]$. This expression vanishes only if we impose the condition that

$$
\Delta_{t} F_{k i}=0 .
$$

This property is true if we explicitly use the definition (28) of $B_{i}=\partial_{i} \Lambda$, but it has to be set as an additional constraint in the algebra generated by the $A_{i}$, the $B_{i}$ and their derivatives. In other words, such an algebra is not free. The homotopy operator $K_{t}$ can be defined only on $B$. In order to solve this problem, we can add to the right hand side of (27) a term which is zero by the constraint (47), but which makes the $\Delta_{t}$ of it vanish algebraically. For this purpose we can choose e.g. $\frac{1}{2} \theta^{k l}\left\{\Delta_{t} F_{k i} \stackrel{\star}{,} A_{l}\right\}$ and consider the expression

$$
U_{i} \equiv D_{i} \dot{\Lambda}+\frac{1}{2} \theta^{k l}\left(\left\{\partial_{k} A_{i} \stackrel{\star}{,} B_{l}\right\}+\left\{\Delta_{t} F_{k i} \stackrel{\star}{,} A_{l}\right\}\right) .
$$

Then

$$
\Delta_{t} U_{i}=0
$$

algebraically and we can apply the homotopy operator to $U_{i}$ and obtain (31). This is the same procedure we have proposed in [2] and [3] to treat the analogous difficulty.

\section{Solutions to higher order in $\theta$}

Observe that we can recover the first order in the $\theta$ expansion as

$$
\begin{aligned}
& \Lambda^{(1)}=\left.\dot{\Lambda}(t)\right|_{t=0}=\frac{1}{4} \theta^{i j}\left\{\partial_{i} \lambda, a_{j}\right\}, \\
& A_{i}^{(1)}=\left.\dot{A}_{i}(t)\right|_{t=0}=-\frac{1}{4} \theta^{k l}\left\{a_{k}, \partial_{l} a_{i}+f_{l i}\right\},
\end{aligned}
$$

which yields the well-known solution found by Seiberg and Witten [1]. More in general, once we have the expressions (30) and (31) to first order, the evolution equations provide a useful method for computing the terms of higher order in $\theta$ by just noticing that

$$
\Lambda^{(n)}=\left.\frac{1}{n !} \frac{\partial^{n} \Lambda(t)}{\partial t^{n}}\right|_{t=0}, \quad A_{i}^{(n)}=\left.\frac{1}{n !} \frac{\partial^{n} A_{i}(t)}{\partial t^{n}}\right|_{t=0} .
$$

Therefore, by simply differentiating with respect to $t$, we can compute $\Lambda^{(n)}$ and $A_{i}^{(n)}$. This is an alternative and easier technique than applying the homotopy operator order by order as suggested in $[2,3]$.

In particular to second order we get

$$
\begin{aligned}
\ddot{\Lambda}= & \frac{1}{4} \theta^{k l}\left(\left\{\partial_{k} \dot{\Lambda}^{\star}, A_{l}\right\}+\left\{\partial_{k} \Lambda \stackrel{\star}{,} \dot{A}_{l}\right\}+\partial_{k} \Lambda \dot{\star} A_{l}+A_{l} \dot{\star} \partial_{k} \Lambda\right) \\
\ddot{A_{i}}= & -\frac{1}{4} \theta^{k l}\left(\left\{\dot{A}_{k}^{\star}, \partial_{l} A_{i}+F_{l i}\right\}+\left\{A_{k} \stackrel{\star}{,} \partial_{l} \dot{A}_{i}+\dot{F}_{l i}\right\}\right. \\
& \left.+A_{k} \dot{\star}\left(\partial_{l} A_{i}+F_{l i}\right)+\left(\partial_{l} A_{i}+F_{l i}\right) \dot{*} A_{k}\right) .
\end{aligned}
$$


Notice that the equation for $\frac{\partial^{n} \Lambda}{\partial t^{n}}$ contains $\frac{\partial^{n-1} A_{i}}{\partial t^{n-1}}$, while the equation for $\frac{\partial^{n} A_{i}}{\partial t^{n}}$ depends only on $\frac{\partial^{k} A_{i}}{\partial t^{k}}, k=0, \ldots, n-1$. This means that the equations for $A$ are independent from those for $\Lambda$. We need to compute $A$ first and only afterwards we can substitute it in the expression for $\Lambda$. If we use the homotopy operator, exactly the opposite happens, we need the expression for $\Lambda^{(n)}$ first in order to obtain $A_{i}^{(n)}$. If we insert the expressions (30) for $\dot{\Lambda}$ and (31) for $\dot{A}_{l}$ we obtain

$$
\begin{aligned}
\ddot{\Lambda}= & \frac{1}{16} \theta^{i j} \theta^{k l}\left(\left\{\left\{\partial_{i} \partial_{k} \Lambda \stackrel{\star}{,} A_{j}\right\}+\left\{\partial_{i} \Lambda \stackrel{\star}{,} \partial_{k} A_{j}\right\} A_{l}\right\}\right. \\
& -\left\{\partial_{i} \Lambda^{\star},\left\{A_{k} \stackrel{\star}{,} \partial_{l} A_{j}+F_{l j}\right\}\right\} \\
& \left.+2 i\left[\partial_{i} \partial_{k} \Lambda \stackrel{\star}{,} \partial_{j} A_{l}\right]\right) .
\end{aligned}
$$

\section{Ambiguities}

The solution (55) has to be compared to other known solutions of the SW map at the second order, like e.g. [4] or [6]. Before doing that, let us remark that the solutions of (26) and (27) are not unique. This has been commented on by a number of authors $[2,3,4,6,9,14]$.

If we start with the structure equations (9), (10)

$$
\begin{aligned}
s \Lambda & =i \Lambda \star \Lambda, \\
s A_{i} & =\partial_{i} \Lambda-i\left[A_{i}, \Lambda\right],
\end{aligned}
$$

and consider a change in $\theta$ by an amount $\delta \theta$, then we see that

$$
\Delta \delta \Lambda=-\frac{1}{2} \delta \theta^{k l} \partial_{k} \Lambda \star \partial_{l} \Lambda
$$

where the star product and the fields are at $t=1$ and where $\Delta$ is the same as $\Delta_{t}$ for $t=1$. Therefore, given a solution $(\delta \Lambda)_{0}$ of this equation,

$$
\delta \Lambda=(\delta \Lambda)_{0}+\Delta H
$$

is also a solution, because of the nilpotency of $\Delta$.

Similarly, for the gauge potential a change in $\theta$ induces a change in $A_{i}$ determined by

$$
\Delta \delta A_{i}=D_{i} \delta \Lambda+\frac{1}{2} \delta \theta^{k l}\left\{\partial_{k} A_{i} \stackrel{\star}{,} \partial_{l} \Lambda\right\} .
$$

Therefore, given a solution $\left(\delta A_{i}\right)_{0}$ corresponding to $(\delta \Lambda)_{0}$, the solution corresponding to $(\delta \Lambda)_{0}+\Delta H$ is

$$
\delta A_{i}=\left(\delta A_{i}\right)_{0}+D_{i} H+S_{i}
$$

where $S_{i}$ is an arbitrary local functional of ghost number 0 satisfying

$$
\Delta S_{i}=0
$$


This is a consequence of the fact that $\Delta$ commutes with the covariant derivative: $D_{i} \Delta=\Delta D_{i}$. The ambiguities determined by $H$ are of the form of a gauge transformation.

Due to the definition of $\Delta$ the condition (60) means that $S_{i}$ transforms covariantly

$$
s S_{i}=i\left[\Lambda, S_{i}\right]
$$

This covariant ambiguity is of a different type from the gauge ambiguity. It can be interpreted as a field dependent redefinition of the gauge potential.

The ambiguities of gauge type are an infinitesimal version of the Stora invariance [3] of the structure equations (9), (10)

$$
\begin{aligned}
\Lambda & \rightarrow G^{-1} \star \Lambda \star G+i G^{-1} \star s G, \\
A_{i} & \rightarrow G^{-1} \star A_{i} \star G+i G^{-1} \star \partial_{i} G,
\end{aligned}
$$

where $G$ is an arbitrary local functional of ghost number 0 .

If we compare the solution to second order given in (55) for $t=0$

$$
\Lambda^{(2)}=\left.\frac{1}{2} \ddot{\Lambda}\right|_{t=0}
$$

with the solution $\Lambda^{\prime(2)}$ found in [4] we see that

$$
\begin{aligned}
\Lambda^{(2)}-\Lambda^{\prime(2)}= & \frac{1}{64} \theta^{k l} \theta^{m n} \Delta_{0}\left(\left\{\left\{D_{m} a_{k}+D_{k} a_{m}-f_{k m}, a_{n}\right\}, a_{l}\right\}\right. \\
& \left.-\left[\left[a_{k}, a_{m}\right], f_{n l}\right]\right)
\end{aligned}
$$

which is an ambiguity of the gauge type.

\section{Actions}

Until this point, we have discussed the deformation of gauge structures and their representations in terms of Yang-Mills fields, without any reference to the dynamics of the fields themselves. To specify the dynamics, we must construct actions that are invariant under the deformed gauge transformations

$$
\begin{aligned}
& s A_{i}=\partial_{i} \Lambda-i\left[A_{i} \stackrel{\star}{,}, \Lambda\right], \\
& s F_{i j}=-i\left[F_{i j} \stackrel{\star}{,} \Lambda\right] .
\end{aligned}
$$

The procedure is analogous to the construction of commutative Yang-Mills theory. One arrives at the expression

$$
S^{Y M}[A]=-\frac{1}{4} \int d^{4} x \operatorname{Tr} F_{i j} \star F^{i j},
$$

where $F_{i j}$ is the noncommutative field strength given by (32), and the trace is the ordinary matrix trace in the appropriate representation. The proof of the invariance of (66) under (65) is based on the properties

$$
\begin{aligned}
& \int d x f \star g=\int d x f g=\int d x g \star f, \\
& \int d x \operatorname{Tr} M \star N=\int d x \operatorname{Tr} N \star M,
\end{aligned}
$$


the latter of which is valid for any pair of matrix valued functions, when surface terms are ignored. Hence, the integral of the trace is invariant under any cyclic permutation of its factors, also in the presence of the star product. Since the fields $A$ and $F$ are generally valued in the enveloping algebra, we have to use the Seiberg-Witten map in order to make sense of (66) as a theory with a finite number of degrees of freedom, namely those of $a_{i}$. To first order in the deformation parameter $\theta$, we find

$$
\begin{array}{r}
S^{Y M}=-\frac{1}{4} \int d^{4} x \operatorname{Tr} f_{i j} f^{i j}+\frac{1}{16} \theta^{k l} \int d^{4} x \operatorname{Tr} f_{k l} f_{i j} f^{i j}- \\
-\frac{1}{2} \theta^{k l} \int d^{4} x \operatorname{Tr} f_{i k} f_{j l} f^{k l}+O\left(\theta^{2}\right),
\end{array}
$$

where $f_{i j}$ is the commutative field strength given by (16).

We would like to remark that at the level of free fields there is no difference between commutative and noncommutative theories, because the properties (67) guarantee that the star product disappears from any quadratic action. It is only when interaction terms are present that the commutative and the noncommutative theories are in fact different. However, interaction terms are always present in the action (66), even if the gauge group is $\mathrm{U}(1)$, because of the star commutator term in the expression (32) for $F$.

In addition to the pure Yang-Mills theory, one can construct a noncommutative version of any action with a gauge-invariance, simply by replacing each ordinary product of functions with a star product, leaving the matrix multiplication and the trace unchanged, and finally expanding each noncommutative field by means of the Seiberg-Witten map associated with the deformed gauge structure. In particular, Yang-Mills theories with matter fields in various representations have been considered by several authors.

Another gauge-invariant action that can be constructed in terms of gauge potentials only is the Chern-Simons action in three dimensions. Its deformed counterpart is obtained as described above and is

$$
S_{t}^{C S}[A]=\frac{1}{4 \pi} \int d^{3} x \epsilon^{k l m} \operatorname{Tr}\left(A_{k} \star \partial_{l} A_{m}-\frac{2}{3} i A_{k} \star A_{l} \star A_{m}\right),
$$

where the subscript $t$ refers to the parameter of the evolution equation described in section 3.

If one were to expand (69) by means of the Seiberg-Witten map, one would find that it is in fact identical to the undeformed action [10]. In other words

$$
S_{1}^{C S}[A]=S_{0}^{C S}[a]
$$

This can be proven to hold at all orders in the deformation parameter, by showing instead

$$
\frac{d}{d t} S_{t}^{C S}[A]=0, \forall t
$$

The total $t$-derivative is computed using

$$
f \star \dot{g}=\frac{i}{2} \theta^{k l} \partial_{k} f \star \partial_{l} g
$$


and the evolution equation for $A$

$$
\dot{A_{k}}=-\frac{1}{4} \theta^{r s}\left\{A_{r} \stackrel{\star}{,} \partial_{s} A_{k}+F_{s k}\right\} .
$$

In this context, it is worth noting that the WZW model in two dimensions shares the same property, namely the identity of the actions for the commutative and the noncommutative version [11], and that the WZW model in two dimensions is related to the Chern-Simons action in three.

\section{Concluding remarks}

In this paper we have limited ourselves to gauge theories of the Yang-Mills type and have based our analysis on the structure equations (4) and (5) (which involve BRST transformations) and their deformation. This formulation is sufficient for Yang-Mills theories, but for gauge theories with reducible gauge transformations, such as theories with gauge potentials which are differential forms of degree higher than one, it is appropriate to use the antifield formalism of Batalin and Vilkoviskii (BV). The deformation of the gauge structure should then be studied by defining generalized Seiberg-Witten maps in the context of the BV formalism $[12,13,14]$. The use of the master equation couples intimately the gauge transformations and the dynamics, i.e. the action functional.

The existence of the SW map, together with the understanding of its ambiguities, can be interpreted as a kind of "rigidity" of the structure of the gauge group, analogous to the rigidity of semisimple Lie algebras under smooth deformations of the structure constants: the structure constants can be brought back to their original values by performing a linear transformation on the generators. In the case of gauge groups the deformed structure equations can be reduced to the undeformed equations by expressing the deformed fields (e.g. $A_{i}$ and $\Lambda$ ) as local functionals of the undeformed fields $\left(a_{i}\right.$ and $\lambda$ ). Strictly speaking, we have discussed only infinitesimal gauge transformations in a context in which only the space-time coordinates are deformed. Thus, we have ignored all questions for which the topology of the gauge group may be relevant when the gauge fields are quantized [15].

As explained in the introduction, throughout this paper we have considered the case of $\theta^{i j}$ independent of $x$. Techniques of deformation quantization are available for an $x$-dependent Poisson tensor (see, e.g. [16, 17], and references therein, where general coordinate transformations for quantized coordinates are also studied). It would be interesting to extend to that case the results described in the previous sections.

Recently, several authors have pointed out the analogy of the Weyl-Moyal star product with the associative, noncommutative star product which enters in the formulation of Witten's bosonic open string field theory [18, 19, 20, 21, 22, 23]. It would be interesting if methods of deformation quantization developed in the context of the Seiberg-Witten map, would turn out to be useful in string field theory. 


\section{Acknowledgements}

We are very grateful to S. Schraml and A. Weinstein for many fruitful discussions. This paper is a written version of a talk given by B. Zumino at the Workshop on "Continuous Advances in QCD", Minneapolis, Minnesota, May 17-23, 2002. This work was supported in part by the Director, Office of Science, Office of High Energy and Nuclear Physics, Division of High Energy Physics of the U.S. Department of Energy under Contract DE-AC03-76SF00098 and in part by the National Science Foundation under grant PHY-0098840. B.L.C. is supported by the DFG (Deutsche Forschungsgemeinschaft) under grant CE 50/1-2.

\section{References}

[1] N. Seiberg, E. Witten, String theory and noncommutative geometry, JHEP 9909:032 (1999), hep-th/9908142

[2] D. Brace, B. L. Cerchiai, A. Pasqua, U. Varadarajan, B. Zumino, A Cohomological Approach to the Non-Abelian Seiberg-Witten Map, JHEP 06:047 (2001), hep-th/0105192

[3] D. Brace, B. L. Cerchiai, B. Zumino, Nonabelian Gauge Theories on Noncommutative Spaces, to appear in the proceedings of '2001: A Spacetime Odissey', Michigan Center for Theoretical Physics, preprint UCB-PTH01/29, LBNL-48673, hep-th/0107225

[4] S. Goto, H. Hata, Noncommutative Monopole at the second order in $\Theta$, Phys. Rev. D 62:085 (2000), hep-th/0005101

[5] B. Jurčo, S. Schraml, P. Schupp, J. Wess, Enveloping algebra valued gauge transformations for non-abelian gauge groups on non-commutative spaces, Eur. Phys. J. C17:521 (2000), hep-th/0006246

[6] B. Jurčo, L. Möller, S. Schraml, P. Schupp, J. Wess, Construction of nonAbelian gauge theories on noncommutative spaces, Eur. Phys. J. C21:383 (2001), hep-th/0104153

[7] M. Picariello, A. Quadri, S. P. Sorella, Chern-Simons in the Seiberg-Witten map for non-commutative Abelian gauge theories in 4D, JHEP 0201:045 (2002), hep-th/0110101

[8] B. Zumino, Chiral anomalies and differential geometry, in 'Relativity, Groups and Topology II', Les Houches 1983, B. S. DeWitt, R. Stora (eds.), reprinted in S. B. Treiman, R. Jackiw, B. Zumino, E. Witten, 'Current algebra and anomalies', World Scientific, Singapore (1985)

[9] T. Asakawa, I. Kishimoto, Comments on Gauge Equivalence in Noncommutative Geometry, JHEP 9911:024 (1999), hep-th/9909139 
[10] N. Grandi, G. A. Silva, Chern-Simons action in noncommutative space, Phys.Lett. B507:345 (2001), hep-th/0010113

[11] E. F. Moreno, F. A. Schaposnik. Wess-Zumino-Witten and fermion models in noncommutative space, Nucl.Phys. B596:439 (2001), hep-th/0008118

[12] G. Barnich, M. Grigorev, M. Henneaux, Seiberg-Witten maps from the point of view of consistent deformations of gauge theories, JHEP 0110:004 (2001), hep-th/0106188

[13] G. Barnich, F. Brandt, M. Grigoriev, Seiberg-Witten maps in the context of the antifield formalism, preprint ULB-TH/01-18, hep-th/0201139

[14] G. Barnich, F. Brandt, M. Grigoriev, Seiberg-Witten maps and noncommutative Yang-Mills theories for arbitrary gauge groups, preprint ULB-TH/0214, MPI-MIS-44/2002, hep-th/0206003

[15] A. P. Polychronakos, Seiberg-Witten map and topology, preprint RU-02-4B, hep-th/0206013

[16] A. S. Cattaneo, G. Felder, A Path Integral Approach to the Kontsevich Quantization, Commun.Math.Phys. 212:591 (2000), math-qa/9902090

[17] A. S. Cattaneo, G. Felder, On the Globalization of Kontsevich's Star Product and the Perturbative Poisson Sigma Model, hep-th/0111028

[18] E. Witten, Noncommutative Geometry and String Field Theory, Nucl. Phys. B268:253 (1986)

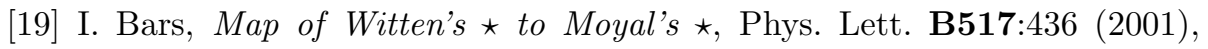
hep-th/0106157

[20] I. Bars, Y. Matsuo, Associativity Anomaly in String Field Theory, preprint CITUSC/02-004, UT-983, hep-th/0202030

[21] I. Bars, Y. Matsuo, Computing in String Field Theory Using the Moyal Star Product, preprint CITUSC/02-013, UT-02-08, hep-th/0204260

[22] M. R. Douglas, H. Liu, G. Moore, B. Zwiebach, Open String Star as a Continuous Moyal Product, JHEP 0204:022 (2002), hep-th/0202087

[23] D.M. Belov, Diagonal Representation of Open String Star and Moyal Product, preprint hep-th/0204164 